\title{
An Open and Modular Approach for a Context Distribution System
}

\author{
Rami Cohen \\ Computer Science Department \\ Technion, IIT \\ The Technion City \\ Haifa, Israel \\ ramic@cs.technion.ac.il
}

\author{
Danny Raz \\ Computer Science Department \\ Technion, IIT \\ The Technion City \\ Haifa, Israel \\ danny@cs.technion.ac.il
}

\begin{abstract}
The rapid growth of wireless and cellular networks, and the high availability of small communication devices such as PDAs, brings us faster than ever to the point where Context Aware Services (CASs) are becoming a commodity. In order to allow fast and efficient development, deployment, and management of such services, a global system that allows the services to gain access to the context information should be created, maintained, and managed.

In this paper, we study the requirements from such a context distribution system. We deal both with the architectural decisions regarding the definition of context items and the way context information becomes available to the CASs, and the algorithmic aspects of disseminating this information. We demonstrate the advantages of the architecture and the proposed information dissemination algorithms by conducting a simulation study under realistic practical assumptions. Our results indicate that a modular approach in which context information is provided in many network locations by brokers through an open simple API is both powerful enough to provide the needed context information, and simple enough to be easily implemented.
\end{abstract}

\section{Introduction}

The market drive for new advanced services in the converged world of wireless voice and data, brings us faster than ever to the point where Context Aware Services (CASs) are becoming a mainstream service and a major source of income for service providers. By their name (and definition see [5]) CASs are services in which the actual result of using them depends on the context. The interest in such services started in the early nineties in the field of Pervasive Computing, where the context was usually associated with the user location and information provided by various sensors (gadgets). In the wireless communication world, services like "where is the nearest Italian restaurant" or "who is next to me" are being offered to cellular customers, and react according to the location of the user (and other users). The convergence of voice and data networks, and the rapid growth of the wireless world create a need for more sophisticated CASs, in which the context is more than just the user location.

Although the concept (and name) of CASs has only been developed in the last decade (starting with the fundamental paper of Bill N. Schilit et all. [13]), Context Aware Ser- 
vices existed in the telecommunication world for a long time. The most basic example is emergency calls. Emergency calls can be viewed as a service in which the user dials a fixed number (911 in the US and 112 in Europe) but the effect is different, depending on the location of the caller, and sometimes the status of the emergency call centers or the local police station, the call is redirected to the appropriate location. Other advanced voice services, such as follow me, can also be viewed in the same way where the result of dialing a number depends on the context, which is the current location of the recipient of the call (according to the information available to the network). An enhancing of these services in the spirit of Pervasive Computing by inserting specific sensors that detect the current location of a person in the building and forward his (her) calls to the phone located in the same room is described in [16]. The emergency call basic service can also be extended in various ways as described in [12].

As for traditional data services, if one considers a very common service like web browsing, in which we would like to connect to the closest replica of the page we are looking for, or to the one with the lowest load, or to the one with a faster response time (i.e., a combination of the other two). If we envision this as normal web browsing that redirects the page request according to the parameters (server load, traffic load) above, then this is also a Context Aware Service, where the context consists of the client's network location, the location of the replicas in the network, the load on each replica server, the network traffic, and the current routing paths. Such a service is much more network oriented, but the main concepts of CAS are still valid.

A more complex example is the "smart follow me email" system. In such a system, the way email is forwarded to the user depends on several parameters. One is the type of device the user is using (PDA, cell phone, laptop, etc.,), the type of connectivity and available bandwidth (GPRS, WIFI, modem, ADSL, etc.,), and also the importance of the information to the person at this particular time. For example, JPEG pictures are not forwarded to the user when using her PDA and a GPRS cellular data connection (due to the large bandwidth required), unless the email contains the map with the driving instructions for the location of the next meeting. In this case the service reacts according to the context, which is in this case composed of: user location, user private information (meeting schedule), email content, connection type, and available bandwidth.

This last example demonstrates the difficulty in developing complex CASs. The context information needed by the service is complex, it comes from different sources, and (at least some of it) is not managed by the service provider. Thus, in order to deploy CASs, one needs a Context Distribution System (CDS) that can collect the required context information and provide it to the service on a "need to know" basis. This paper deals with the requirements from such a system, both from the architectural and the algorithmic points of view.

The first step in designing a Context Distribution System (CDS) is to understand what is context. As illustrated by the previous examples, context can be anything, from a user location, through information regarding a planned meeting, load and available bandwidth information, to weather information. This fact, as already observed by several researchers ([5]), makes it very hard to formally define and capture all possible context information in a single model. 
Clearly, trying to build a general system that can allow arbitrary data structures and support for general query language could be inherently too complex. A better approach that will allow rapid deployment of such a system would be to design a simple yet powerful enough system that can provide the functionality needed. A key observation here is that the services are the main issue here; context information is needed to allow the delivery of CASs. We should then concentrate on the context information needed by the services in order to allow them to react in a "context aware" way.

Thus, not everything is in fact context. An item becomes a context only if it is needed by a service, for example, the temperature in the Greek island of Samos is a piece of information but it will be a context only if a service that depends on it would be created. This means that when the service is created or deployed, there is a stage where information (from sensors, web server, database, client program, etc.,) is declared as "context". In this way, we can identify the "context information" with an entity that produces this information for the context information system (this may be a different entity from the one that measures or generates this piece of information). In a similar way the service logic is the consumer of this context information. A service is being realized by logic that is running in some network locations. This could be centralized or distributed (as explained in the examples above). Note, that in many cases the same context information, produced by a single producer, is needed by many consumers. For example load and bandwidth information or location information, may be needed by many services, thus our system should be able to handle the dissemination of information to a (possibly large) set of consumers in an efficient way.

A Context Distribution System, needs then, to distribute pre-defined items from producers to consumers. This simplifies the demand from such a system; however, we must also consider efficiency and scalability in the design of such a system. On the other hand, some aspects of the system need not to be fully specified in great detail. Such details can be left out for the different implementation of different vendors.

In this paper we design several aspects of an open, modular, scalable system that provides context information to services. First, in Section 3, we study the requirements and basic architecture needed from such a system. In particular we identify the most important features required from a CDS, difine a simple yet powerfull API that allows to interact with the system, and study the impact of the system's design on the ability to efficiently collect and distribute the context information as needed by next generation services in modern converged networks.

Then, in Section 4 we focus on some of the algorithmic aspects involved in efficient distribution of context information. We show that in order to study the efficiency of context distribution as needed by the services, a new model that considers updates of data, in a multicast/unicast environment should be considered. Using this model, we develop several heuristics to decide how to distribute the information. We investigate the performance of our algorithms using a comprehensive simulation study based on realistic assumptions regarding service need for information. We summarize our work and present some conclusions in Section 5. 


\section{Related Work}

Starting with the fundamental paper of Bill N. Schilit et all. [13], a great research effort was devoted to the study of context aware service. Much of the work in this area comes from the field of Pervasive Computing and deals with information provided by various sensors (gadgets). Many of the early paper describe specific projects that demonstrate the development of a specific context aware service. A good survey that covers some of the interesting work in this area can be found in [5].

In contrast, our paper deals with an open, modular, scalable system that provides context information that comes from various sources to the different services. With the exception of the very recent paper by Heinz-Gerd Hegering and Axel Kupper [12], we are unaware of works that deal with the management of context information in a heterogonous environment. In that paper, the authors define what they call "the context value chain", which describes the way context information is gathered, processed, and used to contextualize services. We use the much simpler producer consumer approach, allow producers to create complex context information from simple (raw) information, and avoid the need to model that process.

The problem of defining and modeling context elements had retained a considerable amount of interest. As observed by many researchers (see [5]) almost any information can affect some service and thus context information can be anything. One way to go in order to model it, is to build a very simple structure in which each context element is just a simple data item specified by its name and its value (key-value pair). Such an approach was taken for example by [14] and [15]. A different approach is to try and build a much more complex model and to allow the customers of the context distribution system to ask complicated queries in a full power query language. Such an approach was taken for example in [11]. We adopt an intermediate approach in which a context items is a result of a fixed predefined query, with a predefined set of parameters (as described in Section $3)$.

\section{Architecture}

As explained before, we are interested in a system that offers CASs that can interact with the network layer. The logic of such a service can be located at a specific network location (say a certain server), or it can be distributed among several network locations.

If we go back to the emergency call example, then we could consider a centralized service, locating at the network main hub, in which a decision regarding the routing of emergency calls in each switch is made (off line), and the resulting instructions are loaded into the different switches. Now, when an emergency call arrives at a certain switch it is always directed to the same location. However, one can consider a distributed implementation (similar to what is called Intelligent Network in the PSTN) in which a local copy of the service is being executed at each switch, and decides (in real time) how to redirect the emergency call. In both cases, the service logic (can also be termed PoP Point of Present) needs an access to the context information - in this case the location from which the call has originated (this information is obtained using the id of the certain switch), and infor- 


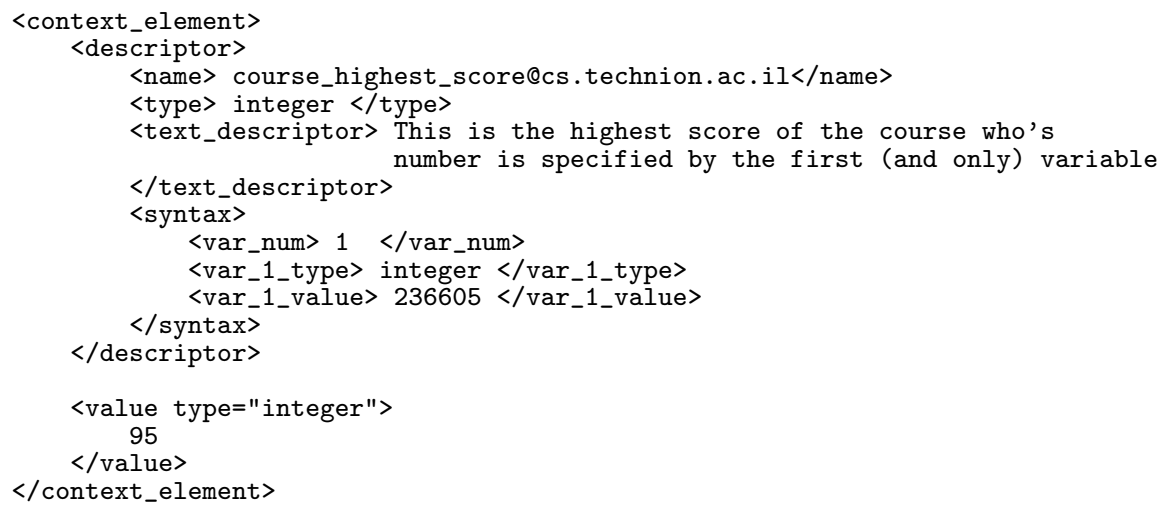

Figure 1: An example of a basic context element.

mation regarding emergency centers in the area. Historically, this information is provided from a proprietary database, specifically created for this service.

In our case we would like to create a general system that can support a variety of CASs and thus we need to consider the variety of the possible services and the different context information each such service may require. Consider again our example regarding web browsing and replicas. As in the previous example, also in this case, one can consider a centralized system that decides which is the best replica, and then redirects the HTTP request to this location (this can be done using the DNS protocol). On the other hand, one can also envision a distributed system (similar to a transparent proxy system) in which the HTTP requests are captured, transparently to the user, and are redirected to the "good" location according to some local logic.

The information needed in this case is more complicated; one needs to know the location of all the replicas of the information requested, and what is the load on each of the servers that have these replicas. A more challenging aspect is the traffic load part. In order to know what is the network load on the path from the customer to each of the possible servers, one needs to know the routing scheme, and the load on each of the links on the path. Collecting and distributing this information is much more complicated. However, most of the routing paths, and the load on many links are not needed by services, so a CDS should not (for scalable reasons) address such information.

We can now discuss what is a context element. One way to go is to build a very simple structure in which each context element is just a simple data item specified by its name and its value (key-value pair). Such an approach was taken for example by [14] and [15]. A different approach is to try and build a much more complex model and to allow the customers of the context distribution system to ask complicated queries in a full power query language. Such an approach was taken for example in [11].

The first observation here is that the first key-value approach could not satisfy the service requirements. If we consider the best replica service, then we would need to have an item in our system for the load on each and every pair of IP addresses, in order to be 


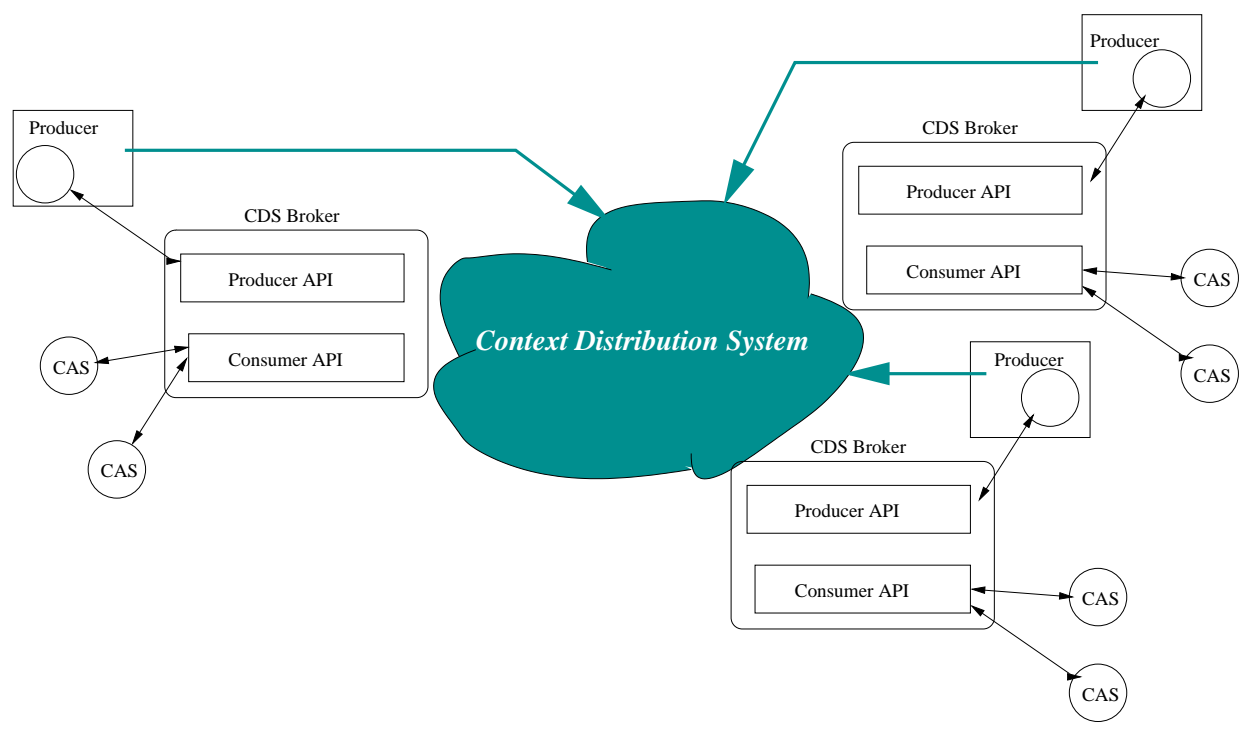

Figure 2: The Abstraction of Context Distribution System and the Broker model.

able to retrieve the load on the path from the client ot each of the possible replicas. This is, of course, not a realistic approach. The second key observation is that the syntax of the needed queries are determined when the service is created. In other words, if a service needs to find the minimal load on the paths between a given IP address, and the IP address of say 5 possible replica locations, then this is fixed and part of the service logic, and will not change. That is, a context item schema for a fixed query can be created and added to the context distribution system when a service is created.

Thus, our solution is as follows. A context item is a result of a fixed predefined query, with a predefined set of parameters. In some cases the parameter list may be empty and then the context item is just a single value. In order to describe the object, we define for each object a description attribute and a value attribute. An XML example of a context item is depicted in Figure 1. Note that a full formal XML Schema for this object can be easily built, but for simplicity we chose to describe here the structure using textual examples. In this example (of Figure 1), we have a context object that has one parameter - the course number. This object returns the value of the highest score achieved in this course. Of course, not all the attributes have to be specified all the time; in particular the text description attributes will not be in every query for that object.

We adopt the provider (some times called producer) consumer approach: Each player in the system is either a consumer of context information - these are the CASs, or a provider of context information. In such architecture, the CDS is responsible to bind the consumers and the producers and to distribute the contexts using a well defined API that will be described letter in this section. Thus, we consider the CDS as a system that has end points (called brokers) at each relevant location. These endpoints can communicate among themselves and produce the information needed. (see Figure 2). Providers can be 


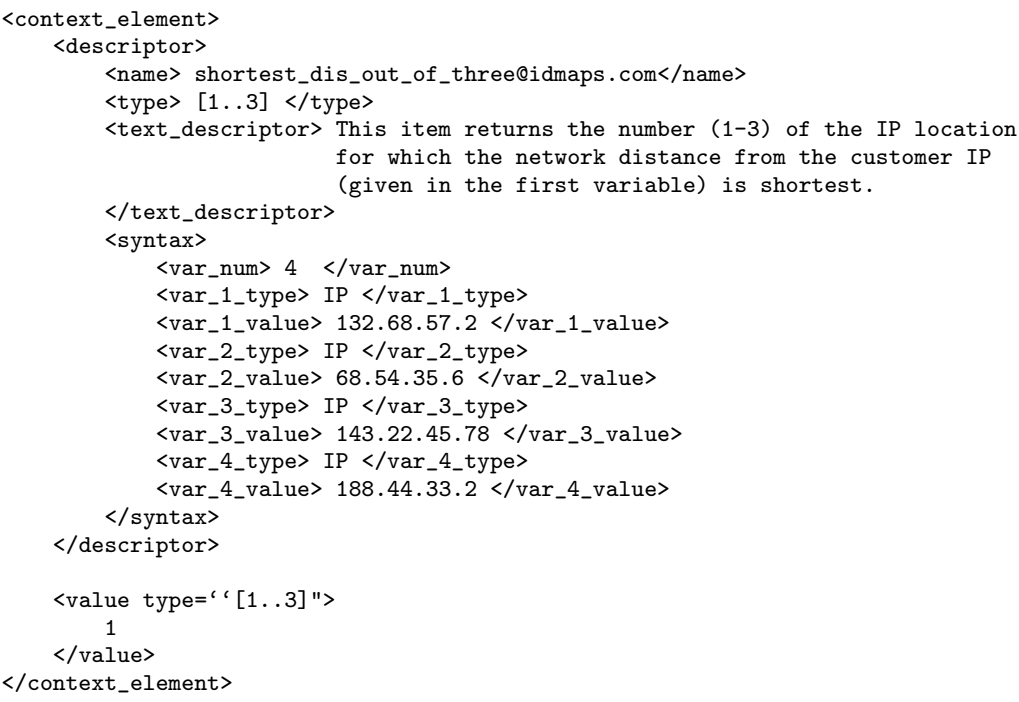

Figure 3: A context element for the closest replica.

special entities that are controlling specific sensors, or processes that rely on information in WebPages, or special processes that produce complex context information from simpler context information available in the system, while consumers are CASs that use these context items for their various services.

Consider the context item described in Figure 3. This is a more complex item that has 4 parameters, the IP address of the client, and 3 IP addresses of servers that have the (web) information requested by this client. The value returned (of type IP address in this case) is the address of the closest (in network terms) server. Such a service is provided by the IDMAPS project [10], thus the CDS could use this service as a context information provider. Now, a combined context provider can be built upon this context item and offer the context item described in Figure 4. This is an object that has two parameters, the IP address of the client, and the web item requested, and it returns the "best" server that has this information. This is a service usually provided by the DNS system, but for a host name and not a URL (i.e. a host name and a specific file name). However, there are proposals that expand the DNS service to handle similar cases (see for example [9]). The term "best" here needs a little clarification, it combines both the load, network distance, and possibly network congestion, in order to decide the location that will allow the best QoS for the client. Providing such a service is far from being easy or trivial, and it is the main business of Content Distribution Network (CDN) Providers like AKAMAI [2]. However, Our open modular architecture allows the easy development and deployment of such information services.

Now we define the API that allows a player to interact with the CDS. Unlike the DNS system, requirements of CASs may be different. In addition to the need for a specific current information, services might be interested in getting a notification regarding changes 


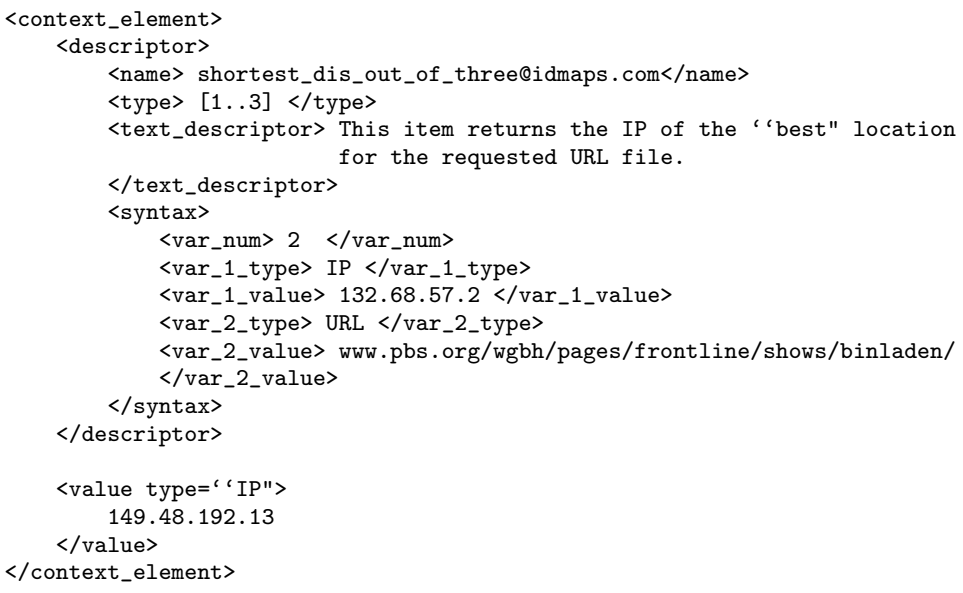

Figure 4: A context element for the "best" replica.

CONSUMER API:

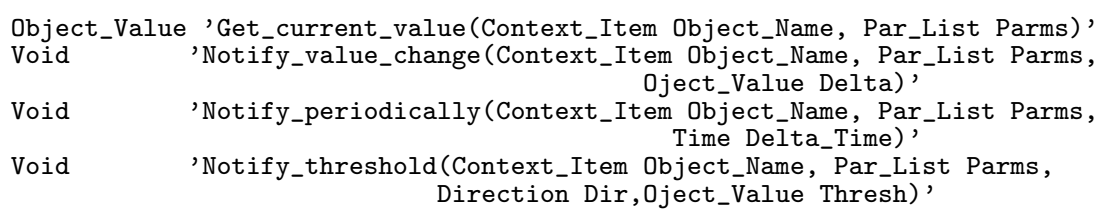

Figure 5: The Context Distribution Broker API.

in the value of a specific item. For example, an emergency center service, needs to be notified when the weather conditions are such that an emergency medical evacuation helicopter cannot be used. It does not make sense to query the weather condition every time period, just to have this updated information, and such a notification service may dramatically reduce the system overhead. The same logic applies to network related services that need to change their behavior upon certain changes in the network load. This type of context information is needed by the CAS on a notification basis.

The API for the CDS Brokers is depicted in Figure 5. The methods in the Consumer API allow a CAS to receive the value of context items. The first method allows a CAS to retrieve the value of a specific context item, by providing its name and parameters. The other three methods allow a CAS to be notified when a certain event occurs. The first one notifies the CAS when a delta change occurs in the value. The second method allows it to be notified periodically, and the third one allows the CAS to be notified when the 
value exceeds (or goes below) a certain predefined threshold. As explained above, and as indicted in [8], such methods are required in order to make the CDS scalable.

The methods in the Producer API, allow a process that delivers context information to introduce the information to the system, and to provide means that will allow the CDS to retrieve the information. The object name is a combination of the producer network address and the local name, as indicated by the names used in the context item examples (see figures 1, 3, and 4). The most involving argument here is the Posible_Access_Methods. This is a list of access methods that can be used by the CDS to retrieve the data. One basic such a method is a web access mechanism in which the context information can be retrieved via a well defined URL composed a specific name and the parameters. Other methods could be used in a way similar to the one adopted by web services [17].

\section{Performance}

In this section we study several ways in which Context Distribution Systems (CDS) disseminate context items to a large number of context consumers (clients). As discussed in the previous section, services can request information using the API defined in Figure 5. There are two basic ways to retrieve information, a regular request for a specific context item (that can be as described before, a result of a complicated predefined query), or a notification request. These two types of access to information are inherently different and we should treat them separately. To simplify the simulation we only deal with "value change notification", but similar results apply to the two other notification methods defined in Figure 5 .

As we mentioned before, we want to abstract away in this research the fine details of the specific implementation of the CDS. Thus we do not consider the exact method the information is retrieved from the provider, and we assume a hybrid scheme where a multicast channel is used in parallel to a unicast dissemination. This way the CDS may reduce its loads by sending a context item to all consumers using the multicast channel instead of sending multiple unicast transmissions.

The scheduling problem using this kind of hybrid scheme has been presented in [7]. In this paper the authors discussed the problem of information dissemination for satellite based Content Distribution Networks (CDNs). They presented a few off-line scheduling algorithms and studied their performances. One big drawback of the model presented in [7] is that updated information is considered a new web item. Thus, the model does not take into account information update. This problem was addressed recently in [6], where the authors expand the model, in a way that can handle retransmission of the same object (when its value is updated). In [6], the computational complexity of the problem is studied, and several off-line scheduling algorithms were presented and theoretically analyzed for worst case performance. However, no practical evaluation of these algorithms is provided, and their theoretical analysis relies on a benefit function that should be derived from the parameters of the needed solution.

In this section we describe a simulation study that evaluates the performances of these off-line scheduling algorithms and compare them to a much simpler greedy on-line algorithm. We assume that the items are in fixed size and one multicast channel is available. 
We divide the time axis into fixed time slots; one item is sent via multicast in each time slot, while all other items are sent via unicast (see [7] ). In order to perform the simulations, we determine that the objective is to reduce the total number of transmission. According to this objective function we compute a benefit function (as needed by the offline algorithm of [6]). Our simulation results show that in many practical scenarios the performances that are obtained by the on-line algorithm are competitive comparing to the off-line algorithms.

As mentioned above, we distinguish between the two ways a CDS can disseminate context information. In the first case the CDS sends an item upon receiving a specific request from the consumer (request item). We assume that each item has an expiration date or an aging attribute that determines the period of time in which a specific copy of the item is valid. This way a consumer can avoid requesting an item as long as it holds a valid copy of that item. In the second case the CDS initiates a transmission of an item to the consumer upon a predefined notification event. This kind of events usually indicate a change in the value represented in the item (e.g. change in the temperature).

Although in both cases the CDS disseminate the same item, their influence on the load and the utilization of the multicast channel in different. In the first case the requests are received from independent consumers. In addition, if the CDS sends a new copy of an item to a consumer, this copy is valid for period of time in which the consumer avoids from requesting this item again. In this case, sending an item via multicast is beneficial since it reduces the necessity to send the item again for a substantial period of time. On the other hand, when an item needed to be sent due to a notification event, the consumer must receive a new copy of that item (even if it has a relatively fresh copy). In addition, a single event affects all the consumers that have been registered to this event, namely there exists a dependency between the consumers. In this case, sending an item via multicast is beneficial since it reduces the necessity to send the item via unicast to each one of the consumers, but unlike the previous case it does not affect future events. Our simulation results show that these two cases (notification and request) are independent, and the cost of any scheduling algorithm is a superposition of the costs of the two cases separately.

\subsection{Simulation Model}

In our simulation we checked the affect of several system parameters on the performance. The system performance is measured by the number of transmissions it takes the CDS to satisfy all consumers, where the cost of a single unicast transmission is equal to the cost of a single multicast transmission*. We say that the cost of algorithm $\mathcal{A}$ is $c$ if the total number of transmissions using this algorithm is $c$. We say that the benefit of an algorithm $\mathcal{A}$ is $b$ if it reduces the cost (i.e. the number of transmissions) by $b$ (comparing to the case when all the multicast channel is not available).

We check the performance in the following two cases:

1. Without a multicast channel (i.e. when the multicast channel is not available).

2. Using an on-line greedy algorithm. This algorithm sends via multicast the most ben-

* One may consider other schemes in which the cost of the multicast channel is zero (e.g. using out of band prepaid broadcast-based channel such as satellite) or in which the cost of the multicast channel is a function of parameters like distance. 


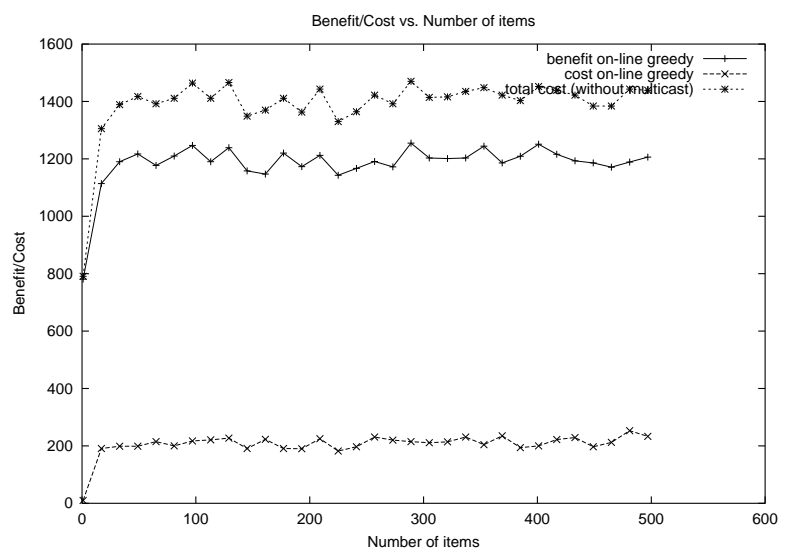

Figure 6: Communication cost vs. the number of items.

eficial item according to the requests and notification events that are gathered during the time slot.

In addition, we also implemented and studied the performances of the off-line algorithms from [6]. These algorithms consider future requests of the items and require offline computation of the benefit function.

In order to simulate practical scenarios we should take into account parameters that may affect the simulation. These parameters include the number of items, the number of consumers, the distribution of requests and notification events, expiration date of items, etc..

In real world implementations, some items are more popular than others. In particular, Zipf distribution is shown to characterize the popularity of the items (see [3] and [4]). In this kind of a distribution, most of the requests and the notification events come from a few items while requests and notifications of all other items are very rare. Hence, the number of effective items in the system is very small, regardless of the total number of items. Figure 6 depicts the cost and the benefit of the on-line greedy algorithm as a function of the number of items. One can see that even for a very small number of items, the benefit from using the multicast channel is clear, and this is the base motivation for using the hybrid scheme where a multicast channel is used in parallel to a unicast dissemination for the CDS. Another observation from Figure 6, is that as excepted, the number of the items does not affect the performance of the algorithm, thus in our simulation we use a small number (15) of items.

Every item has an expiration date or aging attribute that characterize the period of time in which the item is valid. We assume that all the items have the same aging parameter, and we denote by TTL (Time To Live) the number of time slots in which an item is valid.

Next we consider the number of consumers in the simulation and the attributes that are attached to these consumers. In practice, a CDS may serve hundreds or thousands of independent consumers. Each one of these consumers is a CAS that requests different items at a different rate and is registered to different notification events. Although there 

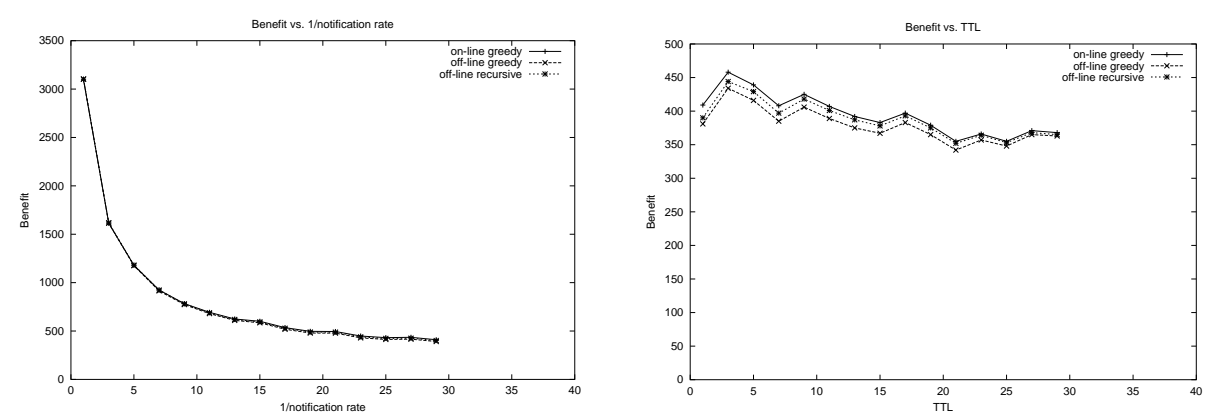

Figure 7: Benefit of the on-line and off-line algorithms

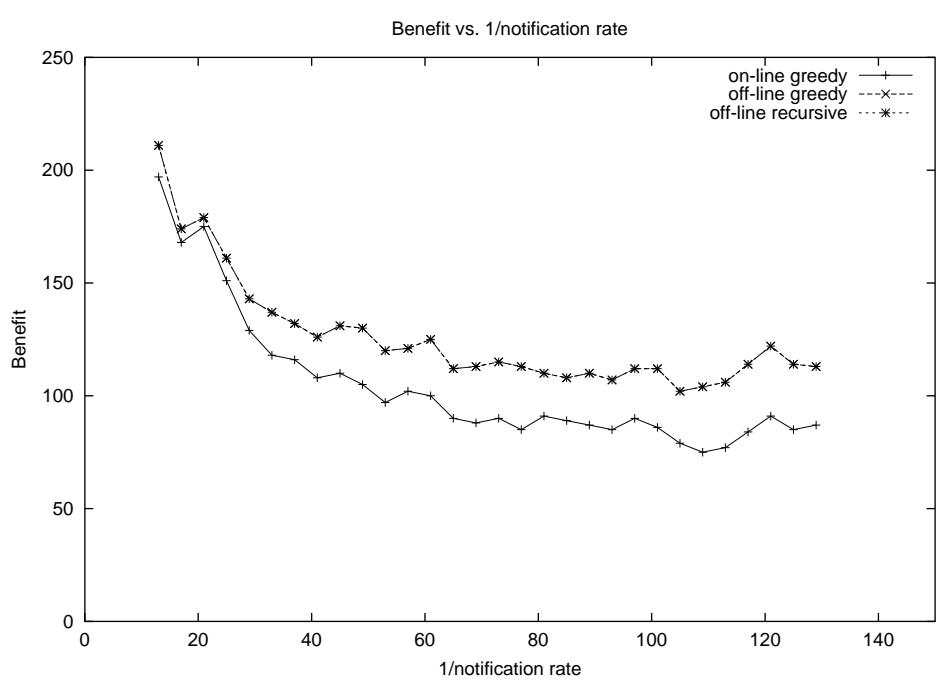

Figure 8: Benefit vs. Notification rate

are many possible configurations, since consumers are independent, a similar behavior will be observed for a reasonable small (100) number of consumers.

We use exponential distribution to characterize the time between two consecutive requests or notification events. We say that the request rate is $r$ if on the average, each consumer requests the most popular item every $r$ time slots. We say that the notification rate is $r$ if in average, the most popular item is being updated every $r$ time slots.

\subsection{Simulation Results}

The main advantage of the off-line algorithms is the capability to prefetch items that may be needed by the consumers relying on the future sequence of requests. Theoretically the performance of the off-line algorithms is bounded while the on-line algorithm does not guaranty a lower bound (see [7]). However, in practical scenarios there are some pa- 
rameters that abolish this advantage and therefore the performance of the algorithms is almost the same. As we mentioned before, the number of effective items is small due to Zipf distribution of the popularity of the items. Hence, the scheduling algorithm does not have many options regarding the item to be sent at each time slot. In addition, notification events do not give any advantage to off-line algorithms since in contrast to other requests they cannot be prefetched. The left part of Figure 7 depict the benefit of the various algorithms vs. the notification rate, and the right part of Figure and 7 depict the benefit of the various algorithms vs. the TTL. Despite the off-line look ahead capability, the on-line greedy algorithm is competitive comparing to the off-line algorithms.

In order to show the benefit of the off-line algorithm we focus in Figure 8 on a scenario where the request distribution changes in time. In this scenario all requests are concentrated in the second half of the simulated interval. Figure 8 shows the benefit of the algorithms vs. the notification rate. In this case the advantages of the off-line algorithms stands out and their performance is much better than the on-line algorithm. As expected, when the number of notification events decrease, the gap between the on-line and off-line algorithms increases.

In the rest of the simulation presented in the section we present only the performances of the on-line greedy algorithm comparing to the maximum cost (i.e. the cost when the multicast channel is not available), using the fact that in practical scenarios the performance of the algorithms is almost identical.

Each notification event causes the CDS to send an item to a large number of consumers. In this case the multicast channel is very beneficial since one multicast transmission saves multiple unicast transmissions. Figure 9 depicts the cost and the benefit and the ratio between the costs vs. the notification rate respectively, in the case where all events are notification events (i.e., there are no item requests). When the number of notification events is large, the probability that more than one notification event will occur in the same time slot is big. In this case only one event can be satisfied using the multicast channel while all other events are satisfied using unicast transmissions. For instance, when the notification rate is equal to 1 time slot, the multicast channel saves $66 \%$ of the transmissions. When the number of notification events decreases, the probability that more than one notification event will occur in the same time slot decreases as well. In this case most of the events are satisfied using the multicast channel. In our simulations the number of consumers is 100 , therefore the multicast channel saves up to $99 \%$ of the transmissions (see the right hand part of Figure 9). Nevertheless, in this case the load over the CDS is very small even without the multicast channel due to a small number of events as indicated by the left hand part of Figure 9.

Next we examine the influence of item requests on the system performance, when the system does not contain notification events. We consider two parameters, the TTL and the request rate of the items.

The right hand part of Figure 10 depicts the cost and the benefit vs. the request rate in this case. The left hand part of Figure 10 depicts the cost ratio in this case. Unlike the previous case (i.e. notification events) each request does not affect a large number of consumers since they are independent. Hence, the benefit of the multicast channel becomes clear when the request rate is high. In this case the probability that an item 

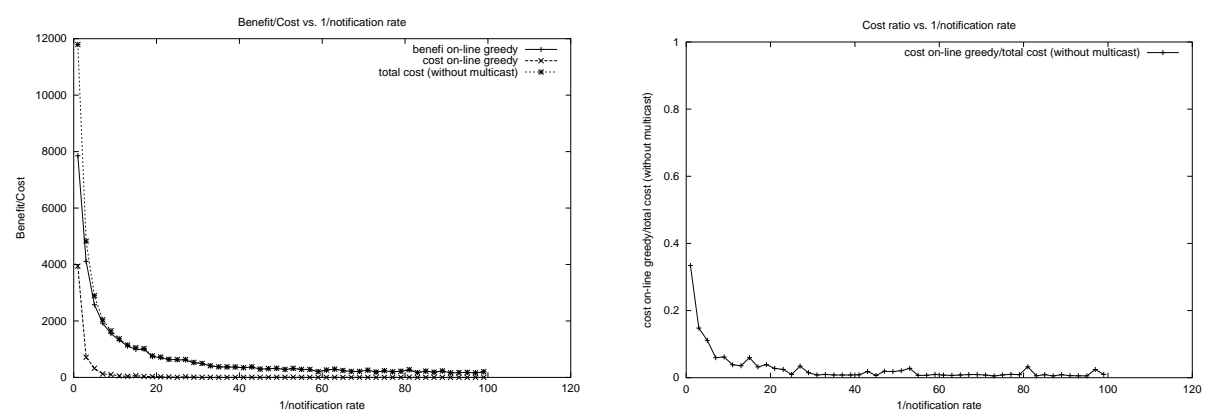

Figure 9: Cost and cost ratio vs. Notification rate
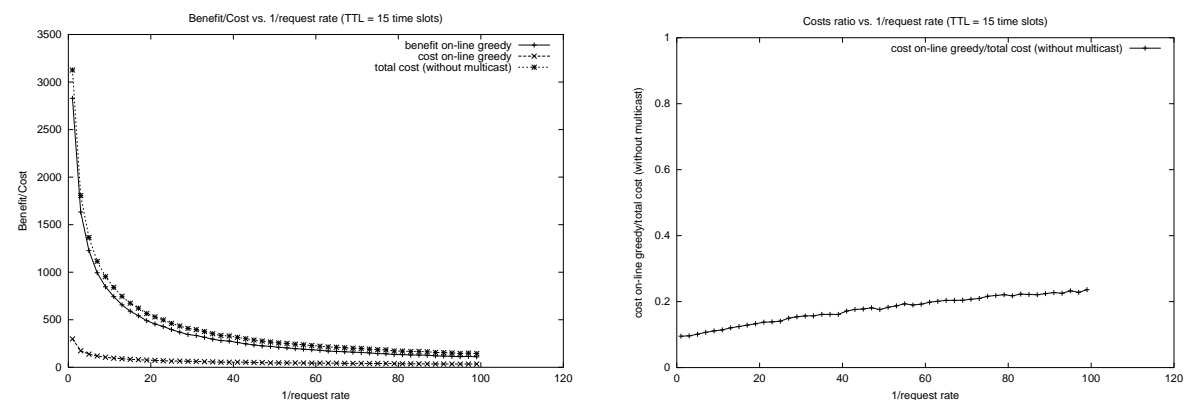

Figure 10: Cost and cost ratio vs. Request rate

will be requested more then once during the TTL period increases when the request rate increases. When there is more than one request during the TTL period the multicast is beneficial and it saves multiple unicast transmissions (see the right hand side of Figure $10)$.

The right hand part of Figure 11 depicts the cost and the benefit vs. the TTL. The left hand part of Figure 11 depicts the cost ratio in this case. When the TTL increases, the cost of the on-line algorithm decreases for two reasons. First, when the TTL is small, the capability to prefetch items decreases, therefore the benefit of the multicast channel becomes smaller (see the left hand part of Figure 11). Second, increasing the TTL increases the time period in which requests of the same item are satisfied; therefore the CDS receives fewer requests.

In practice, a CDS should satisfy both item requests and notification events. Since these two cases are independent by their influence on the CDS (see previous section), the performance of a system is almost a superposition of the system performance of each one of them. Figure 12 depicts the cost and the benefit vs. TTL where the notification rate is equal to 29 time slots and the contribution of the notification events to the total cost (i.e., 

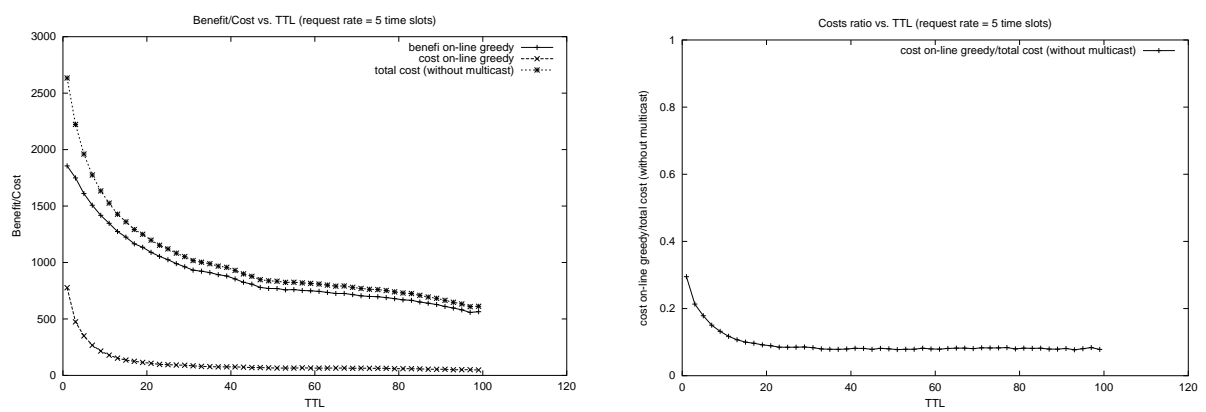

Figure 11: Cost and cost ratio vs. TTL

the cost without using the multicast channel) is 530 while its contribution to the benefit is eight*.

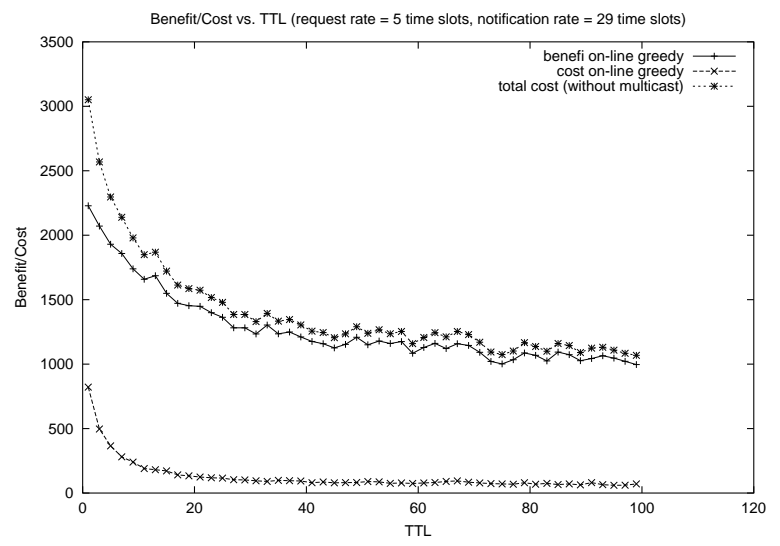

Figure 12: Cost/Benefit vs. TTL

\section{Conclusion}

The contribution of this work is two folded. First we study the requirements and basic architecture needed from an open, scalable and modular Context Distribution System. In particular we identify the most important features required from such a system, and we study the impact of the system design on the ability to efficiency collect and distribute the context information as needed by next generation services in modern converged networks.

Then we focus on some of the algorithmic aspects involved in efficient distribution of context information. We show that in order to study the efficiency of context distribution in the way needed by the services, a new model that considers updates of data, in a mul-

*these numbers have been extracted from the data of Figure 7 
ticast/unicast environment should be considered. Using this model, we develop several heuristics to decide how to distribute the information. We investigate the performance of our algorithms using a comprehensive simulation study based on realistic assumptions regarding service need for information.

In particular we show, that using our designed architecture and our distribution algorithms, one can build an efficient modular open Context Distribution System, that can enable the fast and easy deployment of Context Aware Services, in modern networks.

\section{Acknowledgement}

We would like to thank the members of the CONTEXT IST [1] project (especially WP 3) for many helpful discussions and comments.

\section{References}

[1] Active Creation, Delivery and Management of Efficient Context Aware Services. http://context.upc.es/.

[2] Akamai. http://www.akamai.com.

[3] A. Bestavros. Speculative data dissemination and service to reduce server load, network traffic and service time for distributed information systems. In Proceedings of ICDE'96: The 1996 International Conference on Data Engineering, New Orleans, Louisiana, Mar. 1996.

[4] L. Breaslau, P. Cao, L. Pan, G. Phillips, and S. Shenker. Web caching and Zipf-like distributions: Evidence and implications. In IEEE INFOCOM'99, pages 126 - 134, Mar. 1999.

[5] G. Chen and D. Kotz. A survey of context-aware mobile computing research. Technical Report TR2000-381, Dept. of Computer Science, Dartmouth College, November 2000.

[6] R. Cohen, , D. Raz, and D. Rawitz. Time dependent multi scheduling of multicast. submitted, 2003.

[7] R. Cohen, L. Katsir, and D. Raz. Scheduling algorithms for a cache pre-filling content distribution network. In 21st annual joint conference of the IEEE and communication societies (IFNOCOM), 2002.

[8] M. Dilman and D. Raz. Efficient reactive monitoring. IEEE Journal on Selected Areas in Communications (JSAC), 20:668 - 676, May 2002.

[9] DNS LOC: Geo-enabling the Domain Name System. http://www.ckdhr.com/dnsloc/misc.html.

[10] P. Francis, S. Jamin, C. Jin, Y. Jin, D. Raz, Y. Shavitt, and L. Zhang. "idmaps: A global internet host distance estimation service". IEEE/ACM Transactions on Networking, 9(5):525-540, 2001.

[11] A. Harter, A. Hopper, P. Steggles, A. Ward, and P. Webster. The anatomy of a context-aware application. In Mobile Computing and Networking, pages 59-68, 1999.

[12] H.-G. Hegering and A. Kupper. Management challenges of context-aware servives in ubiquitous environments. Technical report, 2003.

[13] B. N. Schilit, N. Adams, and R. Want. Context-aware computing applications. In IEEE Workshop on Mobile Computing Systems and Applications, Santa Cruz, CA, US, 1994.

[14] B. N. Schilit, M. M. Theimer, and B. B. Welch. Customizing mobile application. In USENIX Symposium on Mobile and Location-independent Computing, pages 129-138, Cambridge, MA, US, 1993.

[15] G. M. Voelker and B. N. Bershad. Mobisaic - an information system for a mobile wireless computing environment. Technical Report TR-95-04-01, 1995. 
[16] R. Want, A. Hopper, V. Falcão, and J. Gibbons. The active badge location system. ACM Transactions on Information Systems, 10:91-102, Jan. 1992.

[17] Web Services Activity. http://www.w3.org/2002/ws/. 